\title{
Inequality in Life Expectancy in Korea according to Various Categorizations of the National Health Insurance Premiums as a Marker of Income
}

\author{
Jinwook Bahk ${ }^{1}$, Hee-Yeon Kang ${ }^{2}$, and Young-Ho Khang ${ }^{2,3}$ \\ ${ }^{1}$ Department of Public Health, Keimyung University, Daegu; \\ ${ }^{2}$ Department of Health Policy and Management, Seoul National University College of Medicine, Seoul; \\ ${ }^{3}$ Institute of Health Policy and Management, Seoul National University Medical Research Center, Seoul, Korea.
}

\begin{abstract}
The purpose of this study was to examine the degree to which the magnitude of income inequality in life expectancy according to different categorization across beneficiary types under the National Health Insurance Service (NHIS) in Korea. We used population and death data in 2017 from the National Health Information Database of the NHIS. Income quintile groups were classified in four ways according to beneficiary type (employee insured, self-employed insured, and Medical Aid beneficiaries). Standard life table procedures were used to calculate life expectancy. The life expectancy gap between the lowest and highest income quintiles was the largest when the entire population was divided into quintiles without distinguishing among types of beneficiaries. In conclusion, we suggest that income quintile indicators in NHIS data, measured without distinguishing among types of beneficiaries, may best represent the magnitude of health inequalities in Korean society. This indicator could be used for future research on health inequalities, as well as for monitoring health inequalities in Korea.
\end{abstract}

Key Words: Life expectancy, National Health Insurance, health policy, socioeconomic factors

In Korea, National Health Insurance Service (NHIS) premiums have been widely used as a marker for income. Many prior Korean studies have examined relationships between NHIS premiums and health indicators, including cancer incidence, screening rates, hospitalization, mortality, life expectancy, and healthy life expectancy. ${ }^{1-11}$ In Korea, NHIS beneficiaries are divided into employee insured (EI) and self-employed insured (SEI) categories. Although NHIS premiums are designed to be imposed according to the income level of the insured, NHIS premiums are calculated differently depending on the beneficiary type.

Received: March 5, 2020 Revised: May 28, 2020

Accepted: June 1, 2020

Corresponding author: Young-Ho Khang, MD, PhD, Department of Health Policy and Management, Seoul National University College of Medicine, 103 Daehak-ro, Jongno-gu, Seoul 03080, Korea.

Tel: 82-2-740-8363, Fax: 82-2-743-2009, E-mail: yhkhang@snu.ac.kr

-The authors have no potential conflicts of interest to disclose.

(c) Copyright: Yonsei University College of Medicine 2020

This is an Open Access article distributed under the terms of the Creative Commons Attribution Non-Commercial License (https://creativecommons.org/licenses/ by-nc/4.0) which permits unrestricted non-commercial use, distribution, and reproduction in any medium, provided the original work is properly cited.
Based on the National Health Insurance Act, NHIS premiums for EI beneficiaries are levied in proportion to monthly income, while premiums for SEI beneficiaries are levied on the basis of income, property, vehicles, and other assets. In addition to health insurance, the Medical Aid system as a public assistance program provides healthcare benefits to low-income families in Korea.

Different methods of classifying income groups have been used in studies employing NHIS premiums as a proxy for income. Some studies have divided individuals into income groups considering the beneficiary type (EI, SEI, or Medical Aid), ${ }^{8-10}$ while others have divided individuals into income groups without distinguishing the beneficiary type. ${ }^{1,2,5}$ The purpose of this study was to examine degrees of variation in the magnitude of inequalities in life expectancy according to NHIS premiums as a marker of income. In doing so, we sought to provide important information on how NHIS premium data should be categorized to set national targets for reducing socioeconomic inequalities in life expectancy and healthy life expectancy in Health Plan 2030.

We used 2017 population and death data from the National Health Information Database (NHID) provided by the NHIS. 
The NHID is linked to mortality registration data from Statistics Korea. ${ }^{12}$ The NHID data cover the whole population of Korea, including NHIS beneficiaries (EI and SEI) and Medical Aid recipients, except for foreigners. According to the National Health Insurance Act, NHIS beneficiaries are Korean nationals who reside within the country, excluding those who receive government-funded medical benefits. Medical Aid recipients were defined as recipients of government-funded medical benefits on the first day of the year based on the Medical Care Assistance Act. Aggregate population and death data without personal identification numbers according to sex, age $(0,1-4,5-9,10-14, \ldots$, $80-84$, and $85+$ years), and income quintiles were obtained from the Big Data Steering Department of the NHIS. Supplementary Table 1 (only online) presents the annual numbers of population and deaths according to sex and income quintiles in 2017. A total of 279487 deaths among 50198087 subjects were analyzed in this study.

NHIS premiums were used as a proxy for income. Prior Korean studies have revealed that the NHIS premiums levied on salaries and household assets are closely associated with mortality and life expectancy. ${ }^{3,11,13,14}$ In this study, we took into account household size, created equivalized household income, and then categorized the equivalized income into quintiles at the nearest quintile points. Medical Aid beneficiaries (MAB, about $3 \%$ of the total population) were grouped into the lowest income quintile, since no premium is levied for MAB. We created four categorizations based on beneficiary type: 1) quintiles for the whole population without distinguishing types of beneficiaries (i.e., combined population of EI, SEI, and MAB); 2) quintiles calculated separately for EI and (SEI+MAB), followed by merging subjects in the corresponding quintile; 3 ) quintiles for the combined population of EI and SEI (i.e., categorization 1, but with MAB excluded); 4) quintiles calculated separately for EI and SEI, followed by consolidating subjects in the corresponding quintile (i.e., categorization 2, but with MAB excluded). Categorizations 1 and 2 included the same populations but differed in the grouping method for income quintiles: in categorization 1, the total population was combined without consideration of beneficiary type, while for categorization 2 , income quintiles were grouped separately for EI and (SEI+MAB) and then the populations in the corresponding quintiles were combined. Categorizations 3 and 4 are versions of categorizations 1 and 2, respectively, with MAB excluded.

Standard life table procedures were used to calculate survival rates. Using the numbers of individual populations and deaths by sex, age group, and income quintile, we constructed abridged life tables using 5-year probabilities of death according to income quintiles from $2017 .{ }^{15}$ We employed the Kannisto-Thatcher method to expand the open-ended age interval 85+ to estimate the probability of dying for 5 -year age groups of 85-89, $90-94, \ldots, 120-124$, and $125+{ }^{16}$ Similar analytic methods have been used in our previous studies. ${ }^{1,25,11}$ Additional analyses of life expectancy for EI quintiles, SEI quintiles, quintiles for SEI+
$\mathrm{MAB}$, and MAB were conducted and presented in Supplementary Table 2 (only online).

This study was approved by the NHIS of Korea (No. NHIS2020-1-097) and the Seoul National University Hospital Institutional Review Board (IRB No. E-2002-016-1098). Individual data linkage was made inside the NHIS at the Big Data Steering Department and aggregate data without any personal information were provided to the authors.

The results of our analysis showed that the life expectancy gap between the lowest and highest income quintiles was largest when quintiles were calculated for the whole population without distinguishing among beneficiary types. The life expectancy difference between the lowest and highest income quintiles was 7.12 years (categorization 1 in Table 1). In contrast, when dividing NHIS premiums into quintiles for EI and (SEI+ $\mathrm{MAB}$ ) separately and then combining subjects in the corresponding quintile [e.g., grouping together the first quintile of EI and the first quintile of (SEI+MAB)], the life expectancy difference between the lowest and highest income quintiles was 5.96 years (categorization 2 in Table 1). When we excluded MAB subjects, the life expectancy difference between the lowest and highest quintiles was smaller than when MAB subjects were included. For the combined population of EI and SEI, the life expectancy difference between the lowest and highest income quintiles was 4.48 years (categorization 3 in Table 1). Meanwhile, when quintiles were divided for EI and SEI separately and then subjects in the same quintiles were combined, the life expectancy difference between the lowest and highest income quintiles was 4.13 years (categorization 4 in Table 1). In categorizations 3 and 4 , the difference in life expectancy between the lowest and highest income quintiles was smaller than the difference in categorizations 1 and 2 because of the exclusion of MAB (one of the most marginalized populations in Korea) from the lowest income group.

In this study, we calculated life expectancy gaps according to income quintiles based on four categorizations using NHIS premiums and the types of beneficiaries. Our analyses showed that the life expectancy difference between the lowest and highest quintiles was largest when income quintiles were grouped for the entire population without distinguishing among beneficiary types. In health inequality research, it is well accepted that the better classification for the social class is achieved when the magnitude of inequality is larger. In the 1910s, social classes officially used in the UK vital statistics were determined based on the gradient of infant mortality. ${ }^{17}$ Similarly, for women, the socioeconomic position indicator resulting in larger mortality inequalities was considered to better reflect socioeconomic environment. ${ }^{18}$

In this study, the magnitudes of life expectancy gaps varied depending on whether $\mathrm{MAB}$ were included in the lowest quintile group. MAB comprise the most marginalized population in Korean society, especially in terms of health, and face a higher risk of mortality than NHIS beneficiaries. ${ }^{19}$ The result of this study 


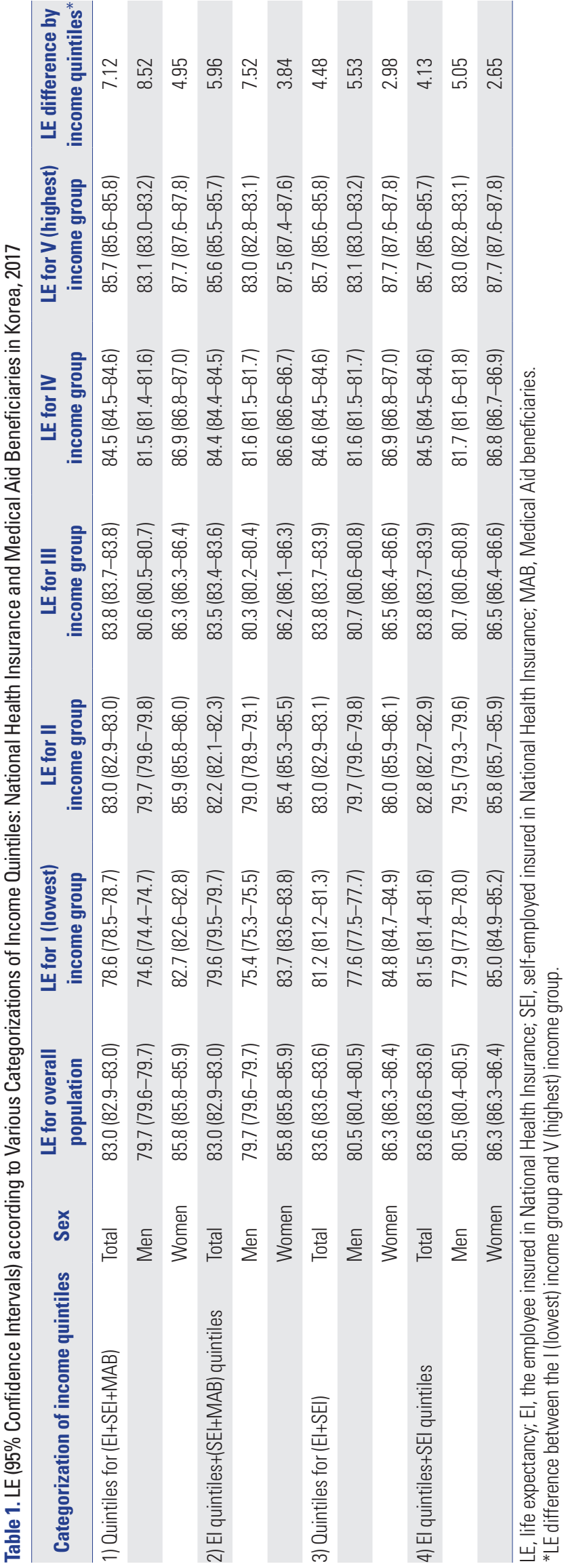

suggests that MAB should not be excluded to show the entire extent of health inequality in Korean society.

The result of this study provide important implications regarding the national monitoring of inequalities in life expectancy by income. The Health Plan 2020 (HP2020) established by the Ministry of Health and Welfare of Korea indicated the necessity of monitoring life expectancy by income to evaluate enhancing health equity, one of the two overarching goals of the HP2020. ${ }^{20}$ The HP2020 also suggested a plan to monitor life expectancy and health expectancy according to income and geography. The HP2020 has presented results on monitoring inequalities in health indicators by income and urbanity. ${ }^{21} \mathrm{~A}$ study conducted by the Korean Society for Equity in Health suggested that health expectancy by income quintiles should be seen as the quantifiable overarching goal for health equity and indicated that the NHID and NHIS premiums could be used for monitoring this indicator. ${ }^{22}$ Health inequalities according to income and geography were also highlighted as measures of the health gap in establishing the Seventh District Health Plan. ${ }^{23}$ Despite the increasing need to monitor inequalities in life expectancy by income, a national consensus has yet to emerge on how to categorize income variables and types of beneficiaries in the NHID. The results of this study indicate that using NHIS premiums as a marker of income without distinguishing among types of beneficiaries might be a simpler and better way to ascertain the largest magnitude of inequalities in life expectancy by income, rather than using both NHIS premiums and beneficiary types. It should be also noted that in 2018, the Korean Society for Equity in Health released the health gap profile for 17 provinces and 252 districts, which employed the NHIS premium quintiles for the combined whole population (without considering types of beneficiaries) as the marker for income. ${ }^{24}$

The results of this study could also be utilized in future Korean studies employing NHIS premiums as a marker of income. Several prior studies analyzing health insurance data used NHIS premiums according to the type of beneficiaries. ${ }^{8-10}$ Both NHIS premiums and beneficiary types are important variables representing indicators of socioeconomic position. Socioeconomic inequalities in health in Korea have been examined according to the NHIS premium ${ }^{1,2,5}$ and the types of beneficiaries. ${ }^{19}$ Both indicators could be used in studies using the NHI premium as a confounder. However, in studies where NHIS premiums are the main variables of interest, it could be used in isolation as a relatively simple marker for income that presents a larger magnitude of health inequalities.

In summary, this study examined variations in the magnitude of inequalities in life expectancy by income in Korea using NHIS premiums categorized into quintile groups as a marker of income. We concluded that the magnitude of the life expectancy gap between the lowest and highest income quintiles was largest when income quintiles were grouped for the whole population without distinguishing among NHIS beneficiary types. The use of NHIS premiums without distinguishing among ben- 
eficiary types may not only be a simple way to monitor inequalities in health by income, but also could be more reflective of health inequalities than methods that consider beneficiary types.

\section{ACKNOWLEDGEMENTS}

This work was supported by a grant of the Korea Health Technology R\&D Project through the Korea Health Industry Development Institute (KHIDI), funded by the Ministry of Health and Welfare, Republic of Korea (grant number: HI18C0446), and a National Research Foundation of Korea (NRF) grant funded by the Korea government (MSIP; number 2017R1C1B5017747).

\section{AUTHOR CONTRIBUTIONS}

Conceptualization: Young-Ho Khang. Data curation: Hee-Yeon Kang. Formal analysis: Jinwook Bahk. Funding acquisition: Young-Ho Khang and Jinwook Bahk. Investigation: all authors. Methodology: Young-Ho Khang and Jinwook Bahk. Project administration: YoungHo Khang. Resources: Young-Ho Khang. Software: Jinwook Bahk and Hee-Yeon Kang. Supervision: Young-Ho Khang. Validation: Jinwook Bahk and Hee-Yeon Kang. Visualization: Jinwook Bahk. Writingoriginal draft: Jinwook Bahk and Young-Ho Khang. Writing-review \& editing: all authors. Approval of final manuscript: all authors.

\section{ORCID iDs}

Jinwook Bahk

Hee-Yeon Kang

Young-Ho Khang

https://orcid.org/0000-0002-7715-9955

https://orcid.org/0000-0001-8530-8087

https://orcid.org/0000-0002-9585-8266

\section{REFERENCES}

1. Khang YH, Lim D, Bahk J, Kim I, Kang HY, Chang Y, et al. A publicly well-accepted measure versus an academically desirable measure of health inequality: cross-sectional comparison of the difference between income quintiles with the slope index of inequality. BMJ Open 2019;9:e028687.

2. Khang YH, Bahk J, Lim D, Kang HY, Lim HK, Kim YY, et al. Trends in inequality in life expectancy at birth between 2004 and 2017 and projections for 2030 in Korea: multiyear cross-sectional differences by income from national health insurance data. BMJ Open 2019; 9(7):e030683.

3. Chun SY, Kim W, Park EC. Disparities in avoidable hospitalization by income in South Korea: data from the National Health Insurance cohort. Eur J Public Health 2019;29:225-31.

4. Ahn C, Hwang Y, Park SK. Predictors of all-cause mortality among 514,866 participants from the Korean National Health Screening Cohort. PLoS One 2017;12:e0185458.

5. Khang YH, Bahk J, Yi N, Yun SC. Age- and cause-specific contributions to income difference in life expectancy at birth: findings from nationally representative data on one million South Koreans. Eur J Public Health 2016;26:242-8.

6. Jung HM, Lee JS, Lairson DR, Kim Y. The effect of national cancer screening on disparity reduction in cancer stage at diagnosis by income level. PLoS One 2015;10:e0136036.
7. Kim JM, Kim HM, Jung BY, Park EC, Cho WH, Lee SG. The association between cancer incidence and family income: analysis of Korean National Health Insurance cancer registration data. Asian Pac J Cancer Prev 2012;13:1371-6.

8. Park JH, Lee JS, Lee JY, Hong JY, Kim SY, Kim SO, et al. Factors affecting national health insurance mass screening participation in the disabled. J Prev Med Public Health 2006;39:511-9.

9. Park JO, Shin SD, Kim J, Song KJ, Peck MD. Association between socioeconomic status and burn injury severity. Burns 2009;35:48290.

10. Kim CW, Lee SY, Moon OR. Inequalities in cancer incidence and mortality across income groups and policy implications in South Korea. Public Health 2008;122:229-36.

11. Lim D, Bahk J, Ock M, Kim I, Kang HY, Kim YY, et al. Income-related inequality in quality-adjusted life expectancy in Korea at the national and district levels. Health Qual Life Outcomes 2020;18:45.

12. Seong SC, Kim YY, Khang YH, Park JH, Kang HJ, Lee H, et al. Data resource profile: The National Health Information Database of the National Health Insurance Service in South Korea. Int J Epidemiol 2017;46:799-800.

13. Jung-Choi K, Khang YH, Cho HJ. Socioeconomic differentials in cause-specific mortality among 1.4 million South Korean public servants and their dependents. J Epidemiol Community Health 2011;65:632-8.

14. Khang YH, Yang S, Cho HJ, Jung-Choi K, Yun SC. Decomposition of socio-economic differences in life expectancy at birth by age and cause of death among 4 million South Korean public servants and their dependents. Int J Epidemiol 2010;39:1656-66.

15. Preston SH, Heuveline P, Guillot M. Demography: measuring and modeling population processes. Malden MA: Blackwell Publishers; 2001.

16. Thatcher AR, Kannisto V, Vaupel JW. The force of mortality at ages 80 to 120. Odense, Denmark: Odense University Press; 1998.

17. Macintyre S. The black report and beyond: what are the issues? Soc Sci Med 1997;44:723-45.

18. Sacker A, Firth D, Fitzpatrick R, Lynch K, Bartley M. Comparing health inequality in men and women: prospective study of mortality 1986-96. BMJ 2000;320:1303-7.

19. Bahk J, Kang HY, Khang YH. Trends in life expectancy among medical aid beneficiaries and National Health Insurance beneficiaries in Korea between 2004 and 2017. BMC Public Health 2019;19:1137.

20. Ministry of Health and Welfare. The National Health Plan 2020. Seoul: Ministry of Health and Welfare; 2011.

21. Korea Health Promotion Institute. Health inequality indicators [accessed on 2020 February 26]. Available at: https://www.khealth. or.kr/board?menuId=MENU00743\&siteId=null.

22. Korean Society for Equity in Health, Ministry of Health and Welfare of Korea. Developing key indicators of health equity and strategies for reducing health disparity in national health plan. Sejong, Korea: Korean Society for Equity in Health and Ministry of Health and Welfare of Korea; 2016.

23. Jeong BG. Policy and regional health planning for reducing regional health inequalities. Health Welfare Policy Forum 2018;260: $50-61$.

24. Korean Society for Equity in Health. Health gap profiles at the national, provincial, district levels. Seoul, Korea: Korean Society for Equity in Health; 2018 [accessed on 2020 April 25]. Available at: http://healthequity.or.kr/bbs/view.php?id=pds_thesis\&page $=1$ \& sn $1=\&$ divpage $=1 \&$ sn $=$ off $\&$ ss $=$ on $\&$ sc $=$ on \&select_arrange=headn um\&desc $=$ asc \&no $=24$. 